\title{
Modeling of Linear Concentrating Solar Power using Direct Steam Generation with Parabolic-Trough
}

\author{
Antoine Aurousseau ${ }^{1,2}$ Valéry Vuillerme ${ }^{1} \quad$ Jean-Jacques Bezian $^{2}$ \\ ${ }^{1}$ Univ. Grenoble Alpes, INES, F-33375 Le Bourget du Lac, France \\ CEA, LITEN, Laboratoire des Systèmes Solaires Haute Température, antoine.aurousseau @cea.fr; \\ ${ }^{2}$ Université de Toulouse, Mines Albi, CNRS, Centre RAPSODEE, France;
}

\begin{abstract}
This papers deals with the Modelica /Dymola modeling of linear concentrating solar power in a parabolictrough experimental loop using direct steam generation. An extensive description of the parabolic collector and the absorber tube models is proposed. First results of the simulation of a clear sky day, with the aim of validating the models, are discussed. Experimental data from the CIEMAT-PSA DISS loop in Almeria, Spain, is used.
\end{abstract}

Keywords: Concentrating Solar Power, ParabolicTrough, Direct Steam Generation, Modeling, ThermoSysPro.

\section{Introduction}

Concentrating Solar Power (CSP), or Solar Thermal Electricity, is a promising technology for renewable electricity generation. In its latest Technology Roadmap report (OECD/IEA, 2014), the International Energy Agency estimates that with appropriate R\&D support, the contribution of CSP to the global electricity production could reach $11 \%$ by 2050 .

Among the several CSP technologies, parabolic- focal line of a parabolic mirror. The process of using water as the heat transfer fluid in the tubes and generating steam for a direct use as the working fluid of a thermodynamic cycle is referred as Direct Steam Generation (DSG). It offers several advantages and has potential cost reduction effects, compared to technologies using other heat transfer fluids and heat exchangers (Eck et al., 2008; Feldhoff, Eck, Benitez, \& Riffelmann, 2009).

The combination of the natural transient condition of solar irradiation and the dynamics induced by the presence of a two-phase flow inside the absorber tubes results in a behavior of the steam generation system that is strongly dynamic. Modeling this behavior at the system scale is useful for the sizing and design of both the solar field and its control system.

This paper presents a model of a parabolic-trough solar field, developed with Modelica on the basis of the ThermoSysPro library, developed by EDF R\&D (ThermoSysPro 2014). In the first section, the Modelica model is presented, with a focus on the parabolic collectors and the absorber tubes, and the second section presents the preliminary simulations carried out to validate the models using the

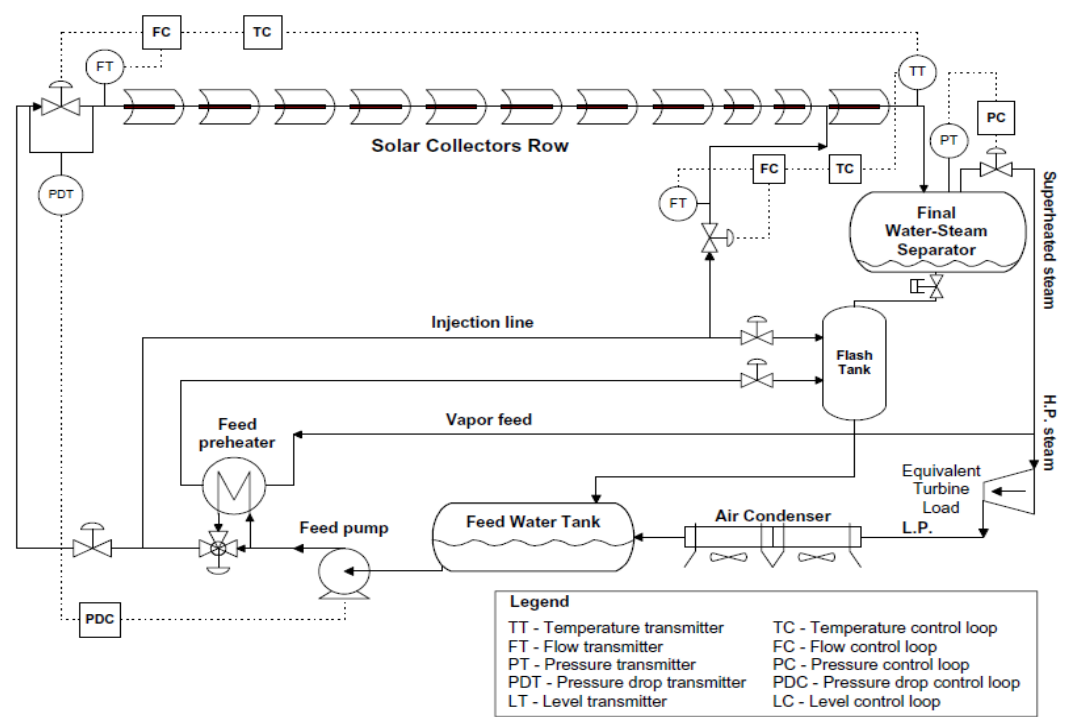

Figure 1: Diagram of the DISS loop (Valenzuela et al. 2005)

trough uses linear concentration to collect heat with a experimental data of the CIEMAT-PSA DISS fluid flowing inside an absorber tube located at the 
experimental loop in Almeria, Spain. Simulations are carried out with the commercial software Dymola.

\section{Models description}

\subsection{The reference experimental setting}

The DISS (for DIrect Solar Steam) experimental loop is located in Almeria, Spain, and is operated by the CIEMAT-PSA institute. It has been operated since about fifteen years, and many studies have been published. It consists in the connection in series of several parabolic-trough collectors, and the appropriate balance of plant installations for the water/steam flow. This study is making use of the experimental loop operated in "once-through" mode, where water is vaporized and steam superheated in the same absorber line, without separation. Description of the experimental setting and collectors details can be found in (Valenzuela, Zarza, Berenguel, \& Camacho, 2004, 2005). Figure 1 shows the experimental loop for the once-through operation mode. It here consists in 11 collectors connected in series, with an injection cooler between the $10^{\text {th }}$ and $11^{\text {th }}$ collector for the control of the outlet steam temperature. Two collectors are 25 meters long and the other ones are 50 meters long.

\subsection{Model structure}

As only the solar field section is modeled (consisting of the 11 connected collectors), presented here is the general structure of a single collector model, consisting of a parabolic mirror and an absorber tube. Figure 2 pictures the structure.

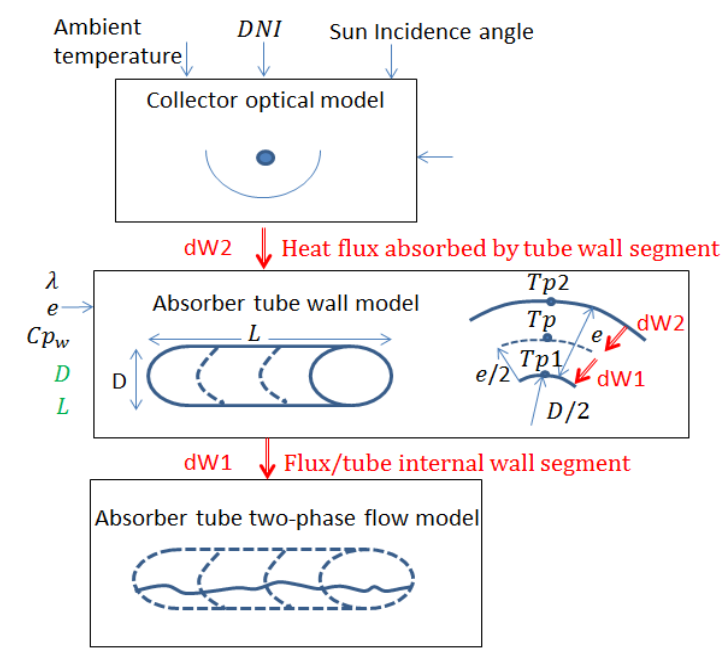

Figure 2: Collector-tube model structure

The optical model computes the heat flux absorbed by the tube wall, then the tube wall model computes the flux through the wall, and the tube two-phase flow model eventually computes the flow conditions. The three "sub-models" are connected with thermal ports and exchange heat flux and temperature data.

\subsection{Collector model}

A LS3-type collector is modeled.

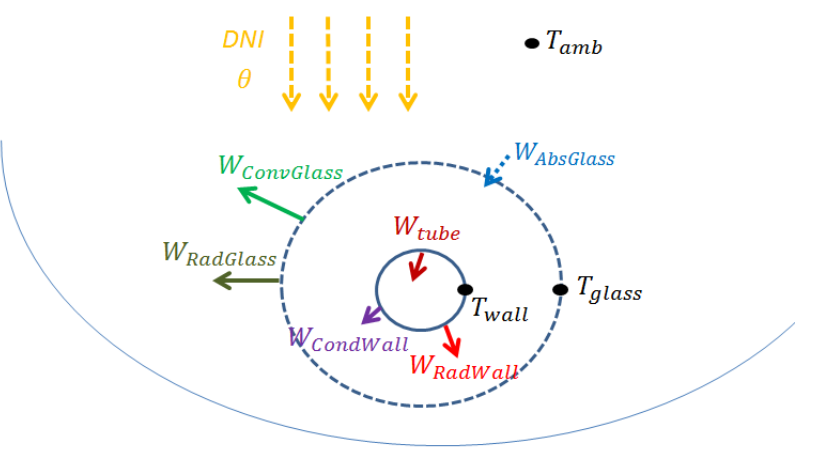

Figure 3: Heat flux diagram on the collector

Figure 3 pictures the collector model in terms of heat fluxes. A developed modified version of the ThermoSysPro 3.1 solar collector is used. The absorber tube and the parabolic mirror are discretized into a defined number of segments, with a set of acausal equations for each of them. The heat flux absorbed by a tube internal wall segment is computed with the following equation:

$$
\begin{gathered}
-W_{\text {tube }}=\eta_{\text {opt }, \text { over }} \times I A M \times \cos \theta \times D N I \\
\times \frac{A_{\text {refl }}}{N_{s}}-W_{\text {RadGlass }} \\
-W_{\text {ConvGlass }}
\end{gathered}
$$

The sign of the heat flux is negative from the parabolic collector point of view, since flux leaving a component is negative by convention. The glass envelope energy balance is computed by the following equation:

$$
\begin{aligned}
d_{M} C_{\text {Pglass }} \frac{d T_{\text {glass }}}{d t} & \\
& =W_{\text {AbsGlass }}+W_{\text {CondWall }} \\
& +W_{\text {RadWall }}-W_{\text {ConvGlass }} \\
& -W_{\text {RadGlass }}
\end{aligned}
$$

Following equations compute the other heat flux terms:

$$
\begin{gathered}
W_{\text {RadWall }}=\frac{A_{\text {tube }}}{N_{s}} \times \sigma \times \varepsilon_{\text {tube }} \times\left(T_{\text {wall }}^{4}\right. \\
\left.-T_{\text {glass }}^{4}\right)
\end{gathered}
$$




$$
\begin{aligned}
& W_{\text {CondWall }}=\frac{A_{\text {Tube }}}{N_{s}} \times \lambda \times\left(T_{\text {wall }}\right. \\
& \left.-T_{\text {glass }}\right) / \frac{D_{\text {tube }}}{2 \log \frac{D_{\text {glass }}}{D_{\text {tube }}}} \\
& W_{\text {RadGlass }}=\frac{A_{\text {glass }}}{N_{s}} \times \sigma \times \varepsilon_{\text {glass }} \times\left(T_{\text {glass }}^{4}\right. \\
& \left.-T_{s k y}^{4}\right) \\
& W_{\text {ConvGlass }}=\frac{A_{\text {glass }}}{N_{S}} \times h \times\left(T_{\text {glass }}-T_{a m b}\right) \\
& W_{\text {AbsGlass }}=D N I \times \frac{A_{\text {glass }}}{N_{s}} \times \alpha_{\text {glass }} \times \cos \theta \\
& \times I A M \times \eta_{\text {opt,Peak }}
\end{aligned}
$$

The equations terms are detailed in Table 1.

\begin{tabular}{|c|l|}
\hline$W_{\text {tube }}$ & Heat flux transmitted by tube wall \\
\hline$W_{\text {RadGlass }}$ & $\begin{array}{l}\text { Heat flux loss through radiation of glass enveloppe } \\
\text { to atmosphere }\end{array}$ \\
\hline$W_{\text {ConvGlass }}$ & $\begin{array}{l}\text { Heat flux loss through convection of glass } \\
\text { enveloppe to atmosphere }\end{array}$ \\
\hline$W_{\text {AbsGlass }}$ & Heat flux absorbed by glass enveloppe \\
\hline$W_{\text {CondWall }}$ & $\begin{array}{l}\text { Conduction heat flux from tube wall to glass } \\
\text { enveloppe }\end{array}$ \\
\hline$W_{\text {RadWall }}$ & $\begin{array}{l}\text { Radiation heat flux from tube wall to glass } \\
\text { enveloppe }\end{array}$ \\
\hline$\eta_{\text {Opt,over }}$ & Overall (glass and tube) collector efficiency \\
\hline IAM & Incidence angle modifier \\
\hline$\theta$ & Incidence angle \\
\hline$D N I$ & Direct Normal Irradiation \\
\hline$A_{\text {refl }}$ & Parabolic mirror aperture area \\
\hline$N_{S}$ & Number of discretization segments \\
\hline$d_{M}$ & Mass of glass enveloppe segment \\
\hline$C_{\text {Pglass }}$ & Glass enveloppe thermal capacity \\
\hline$T_{\text {glass }}$ & Glass enveloppe temperature \\
\hline$A_{\text {tube }}$ & Tube wall heat exchange area \\
\hline$\sigma$ & Boltzmann constant \\
\hline$\varepsilon_{\text {tube }}$ & Tube wall emissivity \\
\hline$T_{\text {wall }}$ & Tube wall temperature \\
\hline$\lambda$ & Inner gas conductivity \\
\hline$D_{\text {tube }}$ & Tube diameter \\
\hline$D_{\text {glass }}$ & Glass enveloppe diameter \\
\hline$T_{\text {sky }}$ & Sky temperature \\
\hline$h$ & Convection heat loss coefficient \\
\hline$T_{\text {amb }}$ & Ambient external temperature \\
\hline$\alpha_{\text {glass }}$ & Glasss absorptivity at normal incidence \\
\hline$\eta_{\text {Opt,Peak }}$ & Peak parabolic mirror optical efficiency \\
\hline & \\
\hline
\end{tabular}

Table 1 : Collector model terms detail

The incidence angle modifier is a function of the incidence angle and is extracted from (Valenzuela et al., 2005) :

$$
I A M=1-0.00188 \times \theta-0.000149206 \times \theta^{2}
$$

\subsection{Two-phase flow model}

A developed modified version of the dynamic twophase flow tube model of the ThermoSysPro 3.1 library is used. Pressure drop correlations were modified from the original version. The two-phase flow tube model is connected to the tube wall model through a thermal port and to other fluid components through fluid ports. The ThermoSysPro structure model and the two-phase flow tube model (highlighted in a circle) are pictured on Figure 4.

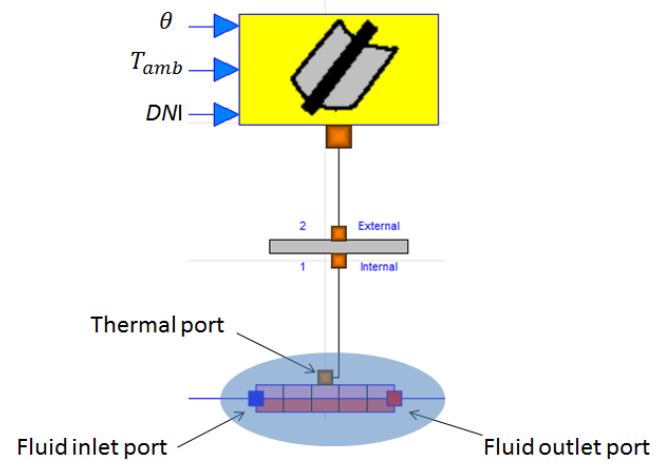

Figure 4: ThermoSysPro collector and tube model diagram

Tubes are discretized only in the longitudinal direction, since ratio between length and diameter is very large. Pressure $P$ and specific enthalpy $h$ are state variables. Mass, energy, and momentum conservation equations, for each i segment of cross section area $A$ yield:

$$
\begin{gathered}
A d x\left(\frac{\partial \rho}{\partial h}_{[i]} \frac{\partial h}{\partial t}_{[i+1]}+\frac{\partial \rho}{\partial P}_{[i]} \frac{\partial P}{\partial t}_{[i+1]}\right) \\
=Q_{[i]}-Q_{[i+1]} \\
\begin{aligned}
A d x\left[\left(h_{i+1} \frac{\partial \rho}{\partial P_{[i]}}\right.\right. & -1) \frac{\partial P}{\partial t} \\
& +\left(h_{i+1+1} \frac{\partial \rho}{\partial h_{[i]}}+\rho_{[i]}\right) \frac{\partial h}{\partial t} \\
& =h_{[i+1]} Q_{[i]}-h_{b[i+1]} Q_{[i+1]} \\
& +d W_{[i]} \\
\frac{1}{A} \frac{\partial Q}{\partial t}_{[i]} d x=P_{[i]} & -P_{[i+1]}-d p f_{[i]}-d p g_{[i]} \\
& -d p a_{[i]}
\end{aligned}
\end{gathered}
$$

With the density $\rho$, pressure $\mathrm{P}$, mass flow rate $\mathrm{Q}$, cell boundary specific enthalpy $h_{b}$, exchanged thermal power $d W$, friction pressure loss $d p f$, gravity pressure loss $d p g$, acceleration pressure loss $d p a$. Dynamics terms in equation (10), like the acceleration pressure term or the inertia term (left hand side) can be set to zero for computations without a full dynamic 
modeling. A Staggered grid is used for the spatial discretization, with the momentum balance equation (10) computed at control volume boundaries.

\subsubsection{Closure equations: pressure losses}

The gravity pressure loss and the acceleration pressure loss terms are computed with homogeneous flow assumptions. The friction pressure loss is computed using separate flows assumption and the MartinelliNelson method: In two-phase flow regions, friction pressure loss is computed as the product of the liquid only-pressure loss and a two-phase flow multiplier:

$$
d p f_{2 \varphi}=\emptyset_{L O}{ }^{2} \times d p f_{L O}
$$

$d p f_{L O}$ is computed as the friction pressure drop with only liquid flowing at full rate, using classical equations. The multiplier $\emptyset_{L O}$ is computed using the Friedel empirical correlation, which was implemented in the model and is considered as the best correlation for this range of mass fluxes:

$$
\emptyset_{L O}{ }^{2}=E+3.24 \times F \times H \times F r^{-0.045} W e^{-0.035}
$$

with

$$
\begin{gathered}
E=(1-x)^{2}+x^{2} \frac{\rho_{l}}{\rho_{g}} \frac{f_{L O}}{f_{G O}} \\
F=x^{0.78}(1-x)^{0.224} \\
H=\left(\frac{\rho_{l}}{\rho_{g}}\right)^{0.91}\left(\frac{\mu_{g}}{\mu_{l}}\right)^{0.19}\left(1-\frac{\mu_{g}}{\mu_{l}}\right)^{0.7} \\
F r=\frac{Q^{2}}{A^{2} \bar{\rho}^{2} g D_{\text {tube }}} \\
W e=\frac{Q^{2} D_{\text {tube }}}{A^{2} \bar{\rho} \sigma_{s}}
\end{gathered}
$$

With $x$ the steam fraction, $\rho_{l}$ the liquid water density, $\rho_{g}$ the steam density.

Liquid-only and steam-only friction coefficients are computed using classical equations involving liquidonly and steam-only Reynolds numbers:

$$
\begin{aligned}
f_{L O} & =\frac{0.079}{R e_{L O}^{0.25}} \\
f_{G O} & =\frac{0.079}{R e_{G O} 0.25}
\end{aligned}
$$

$\mu_{l}$ and $\mu_{g}$ are the water and steam densities, $\sigma_{s}$ the surface tension. $\mathrm{Fr}$ and $\mathrm{We}$ are the Froude and Weber dimensionless numbers. The average density $\bar{\rho}$ is computed the following way:

$$
\bar{\rho}=\left(\frac{x}{\rho_{g}}+\frac{1-x}{\rho_{l}}\right)^{-1}
$$

\subsubsection{Closure equations: heat transfer coefficient}

For each tube segment, the absorbed heat flux is computed with the tube inner wall temperature $\boldsymbol{T}_{\boldsymbol{p}}$ and the fluid segment temperature $\boldsymbol{T}_{\mathbf{1}}$ :

$$
d W=h \times d S \times\left(\boldsymbol{T}_{\boldsymbol{p}}-\boldsymbol{T}_{\mathbf{1}}\right)
$$

The heat transfer coefficient in single-phase flow region is computed using Dittus-Boelter equation:

$$
h=0.023 \frac{k}{D_{\text {tube }}} \operatorname{Re}^{0.8} \operatorname{Pr}^{0.4}
$$

With $k$ thermal conductivity. In two-phase flow region, the heat transfer coefficient is computed using the superposition method of the Chan correlation, described in (Odeh, Morrison, \& Behnia, 1998) :

$$
h_{2 \varphi}=E h_{c l}+S h_{e b}
$$

The single-phase convective boiling term $h_{c l}$ is computed with the Dittus-Boelter equation (22). $E$ is its related corrective term and is computed with a correlation to the Martinelli parameter and the boiling number $B O$ :

$$
E=1+24000 B 0^{1.16}+1.37 X_{t t}^{-0.86}
$$

With $X_{t t}$ the Martinelli parameter:

$$
X_{t t}=\left(\frac{1-x}{x}\right)^{0.9}\left(\frac{\rho_{g}}{\rho_{l}}\right)^{0.5}\left(\frac{\mu_{l}}{\mu_{g}}\right)^{0.1}
$$

$h_{e b}$ is the nucleate boiling contribution term and is computed with an empirical correlation to the ratio of the working pressure to the critical pressure, derived from Stephan and described in (Odeh et al., 1998). The nucleate boiling corrective term $S$ is computed as a function of $E$ and the liquid Reynolds number, an empirical correlation from Gunger \& Winterton and described in (Odeh et al., 1998):

$$
S=1 /\left[1+\left(1.15 E-6 \times E^{2} \times R e^{1.17}\right)\right]
$$

\subsubsection{Closure equations: flow properties}

Flow properties are computed using the IAPWS IF97 water/steam tables and functions. As pressure and enthalpy are computed for each tube segment and each time step, those state variables are used as argument to call properties functions like temperatures, densities, steam fractions, thermal capacities and conductivities, viscosities, etc.

\subsection{Other pressure drops}

Pressure drops outside the collectors, ie. in the connections between them, are modeled with specific 
ThermoSysPro pressure drop components. The experimental pressure data include the loop inlet and outlet pressures, and the pressure drops for each collector. One can then extract the loss induced by the connections between the collectors, and use them to compute pressure drop coefficients to be used in the singular loss models. The coefficient can then be manually adjusted to match the experimental data.

\subsection{Boundary conditions}

The inlet of the collectors line, along with the small injection cooling at the last collector inlet, are modeled as a flow source with imposed mass flow rate values and imposed specific enthalpy values. Those values are directly extracted from the DISS loop experimental data.

The flow outlet of the collectors line is modeled as a pressure sink, with imposed values also directly extracted from the experimental data.

The parabolic collector inputs, direct normal irradiation, ambient temperature, and incidence angle are also directly extracted from the experimental data. As these data are given physical sensors, they require some smoothing with signal processing tools, for the sake of simulations stability.

\section{Simulation of a clear sky day}

A first simulation is carried out with the described model and the input data of a good sunny day of April. Figure 5 shows the measured DNI at the DISS test site on April 22, 2002. Data start at 09:00:00 and the collectors are defocused at 15:56:40, so the simulation is carried out on this time range.

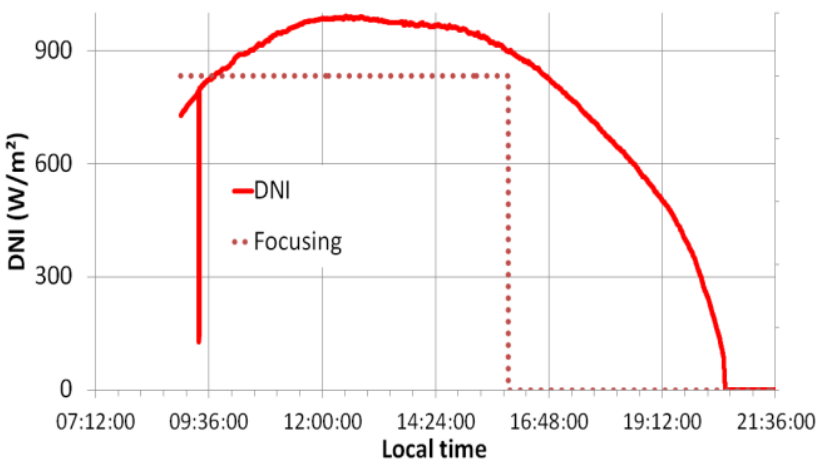

Figure 5: DNI and collector focusing of April 22, 2002

\subsection{Input data for boundary conditions}

\subsubsection{Collector optical model input}

As previously stated, the measured DNI is directly used as input data in the collector optical model. The measured ambient temperature is also used as input to the model, but for the sake of simulation stability, its noisy signal is interpolated with a $5^{\text {th }}$ degree polynomial, as pictured on figure 6 .

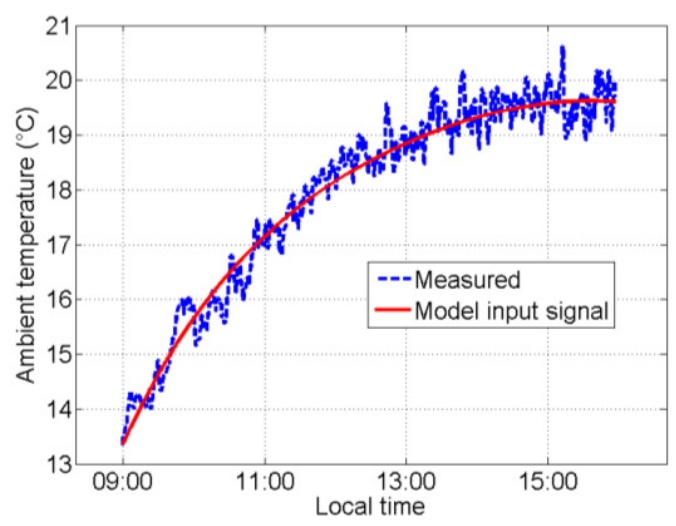

Figure 6: Ambient temperature measured data and model input

The sun incidence angle $\theta$ evolution for April 22, 2002 is extracted from the MeteoNorm database (location: Almeria airport) with an hour time step.

\subsubsection{Flow inlet}

The measured mass flow rate is used as input in the mass flow rate source of the model. The data is processed with a sliding averaging function to smooth the signal, as shown on figure 7 . The test loop is given a temperature setpoint change during the operation, which is why two main evolution sections are visible on the inlet flow rate plot.

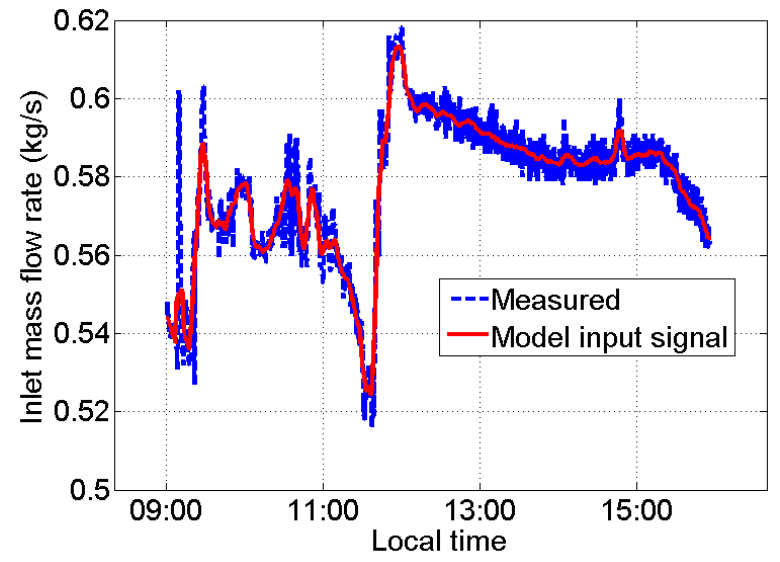

Figure 7: Inlet mass flow rate measured data and model input

For the energy state at the inlet, the ThermoSysPro flow source component requires the specific enthalpy as an input. Available experimental data including temperature and pressure at the first collector inlet, specific enthalpy is computed from those values with the IAWPS IF97 tables, and used as model input.

The injection cooling at the inlet of the last collector, whose role is to keep the outlet temperature on setpoint, is modeled the same way. Its flow rate evolution can be seen on Figure 8 . 


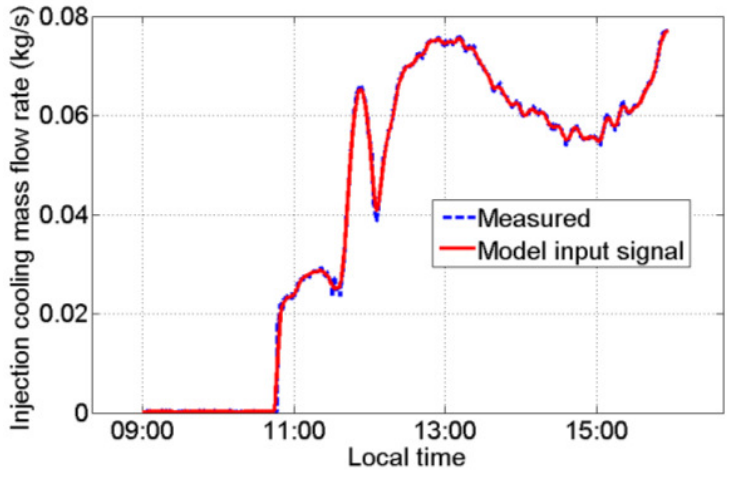

Figure 8: Injected mass flow rate for cooling

\subsubsection{Flow outlet}

The imposed pressure at the collector outlet also comes from experimental data. The outlet pressure control valve is closed until the loop reaches the setpoint pressure at the outlet (loop is then said to operate in sliding pressure mode), then the valve is controlled to keep the pressure at the setpoint (which is an operation in constant pressure mode). For the simulation, no control valve is modeled, and it is simply the outlet pressure that is taken as boundary condition. The pressure field in the loop is then computed from the outlet value and the pressure drops models. As can be seen on Figure 9, the DISS loop is operated to about 31 bars on that day.

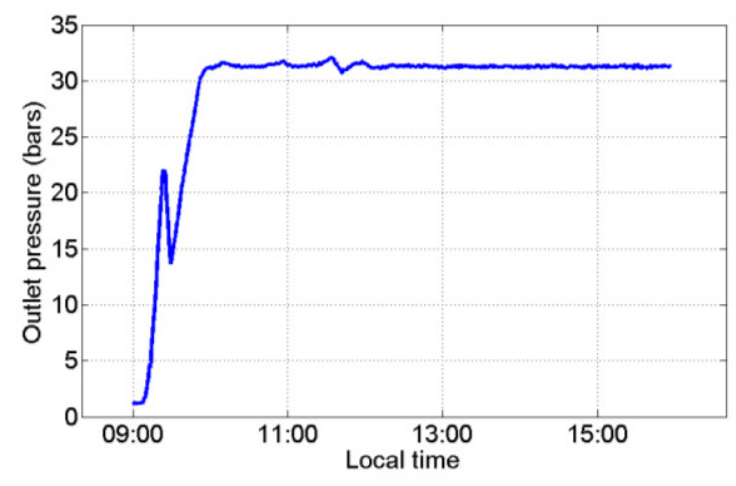

Figure 9: Loop outlet pressure evolution

\subsection{Results and discussions}

\subsubsection{Pressure field in the loop}

Figure 10 shows the pressure at the first collector inlet, thus representing the overall pressure loss in the collector.

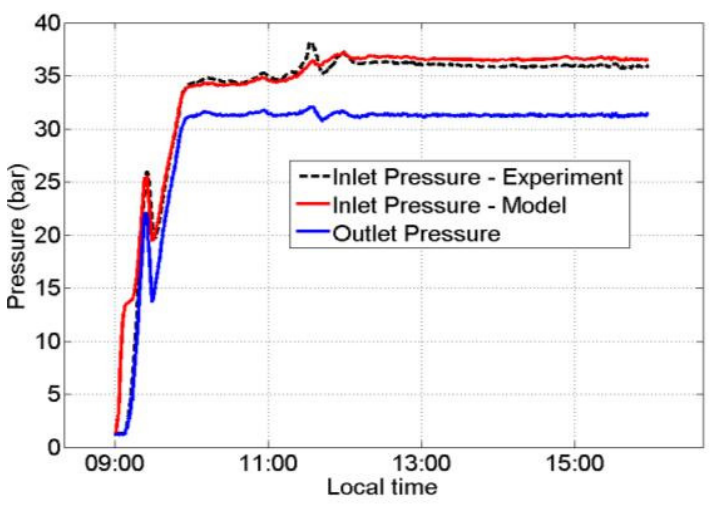

Figure 10: Inlet and outlet pressure in the loop

It can be seen that the computed pressure inlet of the model is quite close to the experimental value, with a small over-prediction of about 1 bar at nominal operation. The dynamic behavior resulting from the change in the boundary conditions is also well described, although the model pressure rises more fastly than the experiment. We assume that this difference is due to the fact that temperatures in the last collectors reach saturation level more fastly in the model (as can be seen in the next section temperature plots), since computation starts with higher enthalpy levels than the experiment (for solver stability reasons). Therefore, if vaporization starts more quickly in the model, a higher pressure drop is observed. The fact that the inertia term of the momentum balance equation (10) is set to zero can also explain this difference between model and experiment, as well as the delay of pressure drop peaks between model and experiment, visible on the following figures.

It seems also interesting to compare specific pressure drops in some collectors. Figure 11 shows the pressure drop inside collectors 1 and 3 . The model clearly under-predicts the pressure loss of collector 1, where flow is only liquid, whereas the prediction is rather good for collector 3, where the flow has two phases. Figure 12 shows the same data for collector 5 and 8 . For those two collectors, where a two-phase flow is present, the model under-predicts the pressure loss. Finally, figure 13 shows the pressure losses for the collectors in the superheating section, collectors 10 and 11 , where superheated steam is found. The prediction for the loss in collector 10 is rather good, whereas a large difference is found with collector 11. This could be explained by the fact that the model does not describe the physics of the injection cooling very well. This phenomenon produces a pressure drop that is not taken into account in the model, which is a simple energy balance flow mixing component. For collector 1 , it is assumed that the large difference between model and experiment is due to the presence of steam bubbles in the first collector of the experimental loop. Indeed, although the average temperature is below saturation, it can locally reach saturation, thus generating small vapor bubbles that will quickly condensate, but will 
generate additional pressure drops. Those bubbles cannot be "seen" by the model, since it uses a homogeneous flow assumption.

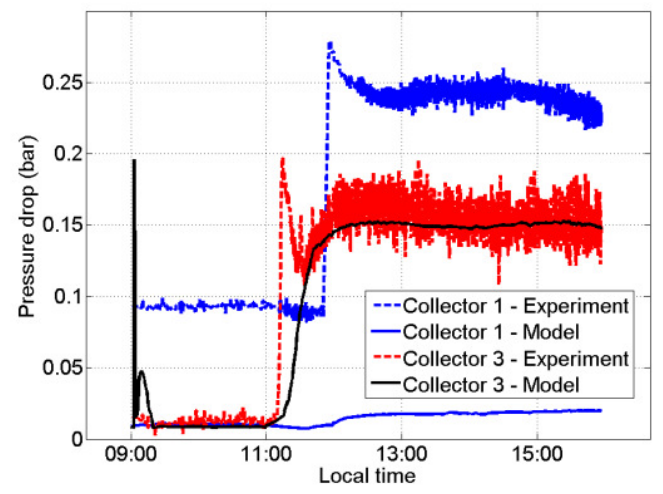

Figure 11: Pressure drop in collectors 1 and 3

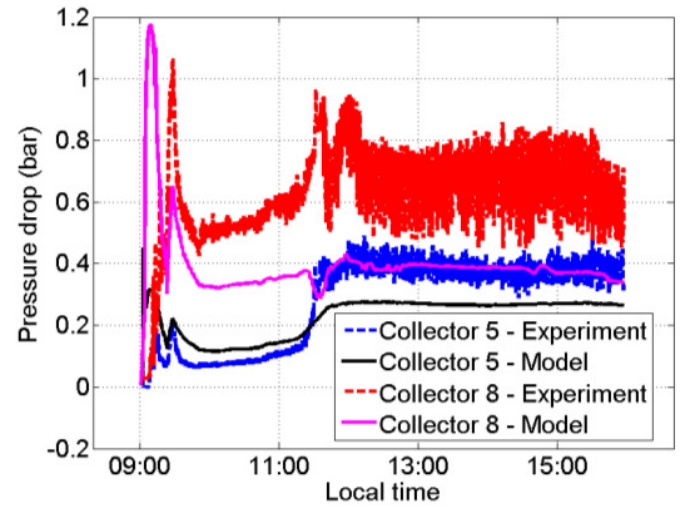

Figure 12: Pressure drop in collectors 5 and 8

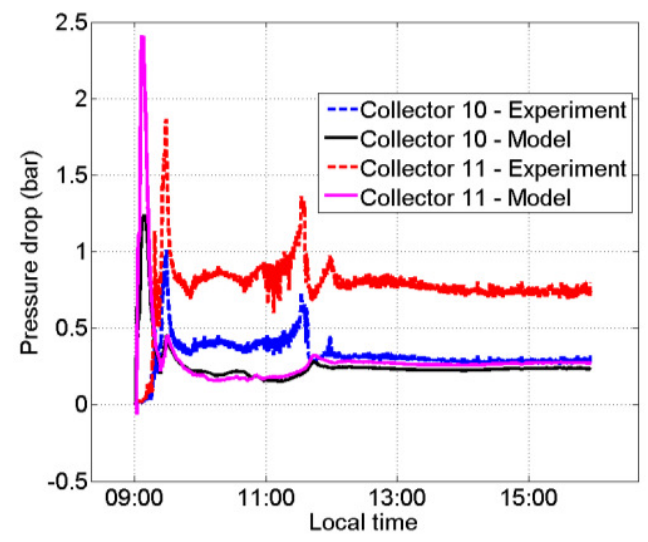

Figure 13: Pressure drop in collector 10 and 11

So it can be seen with the previous figures that although the overall pressure drop along the loop is slightly over-predicted, model pressure losses in each collector are almost always less than experimental data. It is therefore the singular pressure loss components, modelling the connections between collectors, which correct the error. In terms of dynamics, simulation results seems to show a similar behavior as measurements, but smoother.

\subsubsection{Temperatures and steam fractions}

Figure 14 shows the temperature evolution for some of the 8 first collectors. Collectors 2,3,5 and 8 all reach saturation temperature at about $240^{\circ} \mathrm{C}$, which shows that they feature a two-phase flow. The inlet temperature of collector 1 is both the actual experimental value and the model boundary condition. For each of the other collectors, the agreement is good between experimental and modeling values.

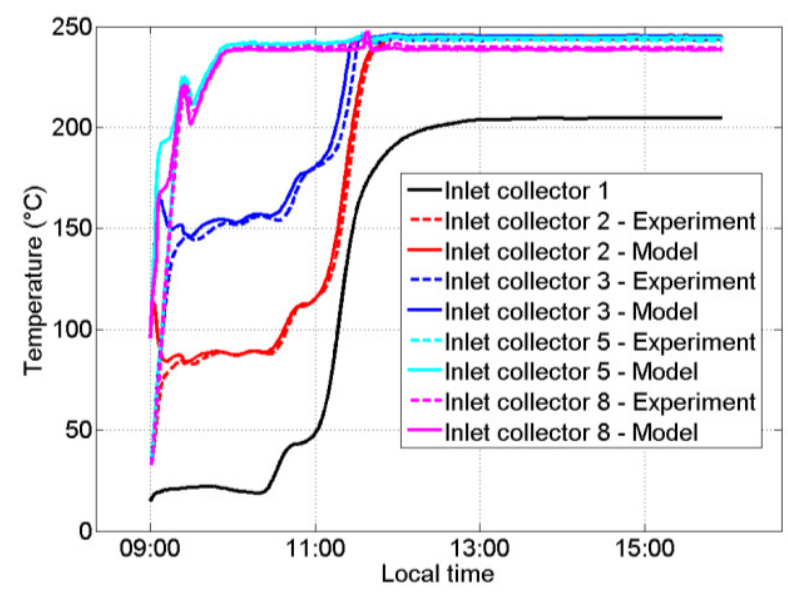

Figure 14: Inlet temperatures of collectors $1,2,3,5$ and 8

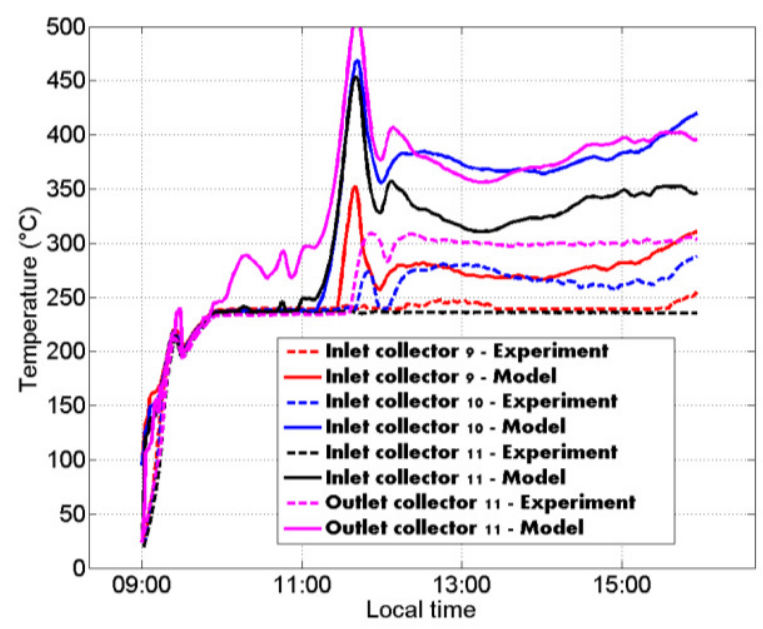

Figure 15: Temperatures of collectors 9 to 11

Figure 15 pictures the temperatures of collectors located in the superheating section of the modeled loop. The model results show indeed that at collector 9 inlet, the temperature is already greater than saturation value. It is not the case for the experimental results, which show that inlet temperature of collector 9 remains at saturation level, although briefly going over it. It means that for the model, superheating starts somewhere in collector 8 . This is confirmed by figure 16 which shows the steam fraction evolution in each of the collector discrete cell. It can be seen that the fraction is 1 from cell 4 on. The experimental results show that inlet temperature of collector 10 is above saturation level, which means that superheating in the experimental loop starts somewhere in collector 9 . 
Therefore there is a significant difference between experiment and model of about one collector's length as to the location of the superheating "beginning". It is also and simply visible by the significant temperature difference between model and experiment for each of the collectors inlets in this superheating section. We assume that this difference comes from the fact that thermal losses are under-evaluated by the parabolic collector model, especially in the superheating section. In particular, the convection losses model uses a fixed heat transfer coefficient, when it actually is a function of many parameters and may be much higher than the value used in the model. Also, the model does not account for the thermal losses in the connections between collectors. Lower thermal losses can also explain the larger temperature peaks visible with the model results. In the experimental loop, larger thermal losses prevent large temperature peaks. Thermal losses can also be under-evaluated in the vaporizer section, but the effect is not visible since temperature remains at saturation value.

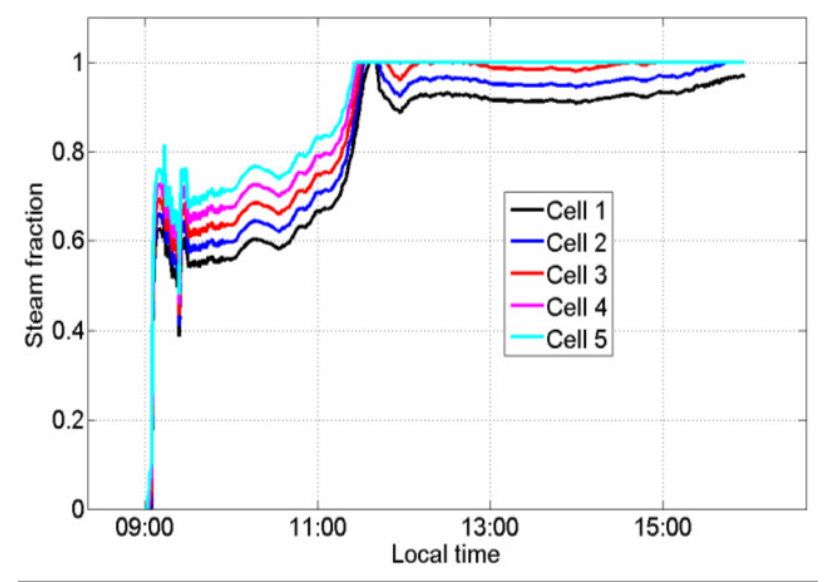

Figure 16: Steam fraction evolution in collector 8

Another source of error is probably the modeling of the injection cooling, or "desuperheating". It is modeled as a simple enthalpy balance component, whereas a complex atomization process actually takes place, with physical phenomenon that are not described by this type of modeling, and which are beyond the scope of this work.

A first general calibration of the model is done by modifying two coefficients. The first one is the modification from the original value of the convection thermal losses coefficient $h$. The coefficient is computed using a free convection correlation (a nowind situation is assumed) extracted from Chan and described in (Forristall, 2003). The second modification is done on the peak optical efficiency of the collectors: it is reduced by 5\%, from $73 \%$ to $68 \%$. As can be seen on the temperature plots of Figure 17, model results are then significantly improved.

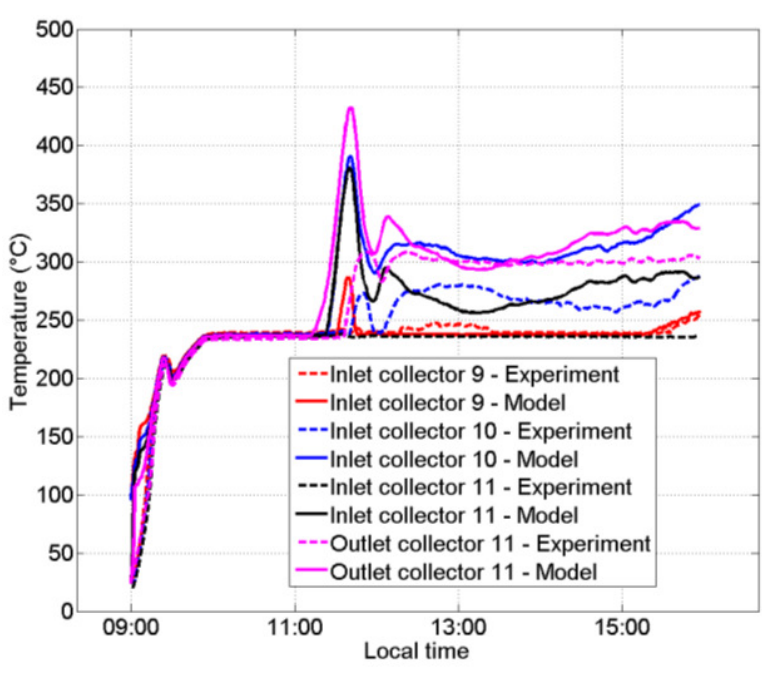

Figure 17: Temperature of collectors 9 to 11, after general model calibration

Better agreement if found for collector 9, the simulated inlet is at saturation temperature. Agreement is also slightly better for collector 10 and at collector 11 inlet, but the difference remains large, in collector 11 in particular. Also, it can be noted that simulated temperatures in collector 11 show transient behaviors that are significantly different from measurements. Since outlet steam conditions are particularly important for DSG systems, it can be stated that the accuracy of those results is not sufficient.

Those remarks, along with previous remarks made about pressure drops, highlight the fact that precise collector-wise calibration should be made in order to improve model performance (indeed, pressure drops coefficients and thermal losses coefficients are assumed to be respectively equal in every collectors, when they are most likely different), and further simulations should be done with a focus on the transient behavior.

\section{Conclusions}

A Modelica model of the direct steam generation parabolic-trough experimental loop DISS has been developed. Simulations have been carried out, using experimental data from the loop as model input and boundary conditions. For the simulated April sunny day, results show a good general behavior agreement between model and experiment, but adjustments have to be made for a better fit to the experimental data, since outlet steam conditions are important in DSG sytems. Special attention will be given to computed flux from the optical model in the parabolic collector model, and to calibration of losses coefficients collector by collector. Also, the simulation of a cloudy day with irradiation transients remains to be done, with full dynamic modeling of the two-phase flow.

The perspective of this work and the validation of the model is the study of advanced control strategies for the handling of irradiation transients, which are key 
to the use of direct steam generation in linear CSP plants. Indeed, knowledge of the dynamics taking place in DSG systems is useful for the parameterization of the control loops.

Simulations with the nuclear two-phase flow code CATHARE are also currently being carried out, for comparison with the Modelica models.

\section{Acknowledgements}

The authors would like to thank their fellow colleagues of CIEMAT for providing the experimental data of the DISS test loop. This collaboration was made possible thanks to the funding from the European Energy Research Alliance (EERA) with the European project N 609837 "Scientific and Technological Alliance for Guaranteeing the European Excellence in Concentrating Solar Thermal Energy - STAGE STE”.

\section{References}

Eck, M., Benz, N., Feldhoff, J. F., Gilon, Y., Hacker, Z., Müller, T., Riffelmann, K.-jürgen, et al. (2008). The potential of direct steam generation in parabolic troughs - results of the German project DIVA. Proceedings of the 14th Biennal CSP SolarPACES Symposium.

Feldhoff, J. F., Eck, M., Benitez, D., \& Riffelmann, K.jürgen. (2009). Economic Potential of Solar Thermal Power Plants with Direct Steam Generation compared to HTF Plants. Proceedings of the ES2009 Conference (pp. 663-671).

Forristall, R. (NREL). (2003). Heat Transfer Analysis and Modeling of a Parabolic Trough Solar Receiver Implemented in Engineering Equation Solver Heat Transfer Analysis and Modeling of a Parabolic Trough Solar Receiver Implemented in Engineering Equation Solver.

OECD/IEA. (2014). Technology Roadmap Concentrating Solar Thermal Electricity.

Odeh, S. D., Morrison, G. L., \& Behnia, M. (1998). MODELLING OF PARABOLIC TROUGH DIRECT STEAM GENERATION SOLAR COLLECTORS. Solar Energy, 62(6), 395-406.

Valenzuela, L., Zarza, E., Berenguel, M., \& Camacho, E. F. (2004). Direct steam generation in solar boilers, using feedback to maintain conditions under uncontrollable solar radiations. IEEE Control Systems Magazine, 1529.

Valenzuela, L., Zarza, E., Berenguel, M., \& Camacho, E. F. (2005). Control concepts for direct steam generation in parabolic troughs. Solar Energy, 78, 301-311. doi:10.1016/j.solener.2004.05.008 